\title{
Perforated Diverticulum of the Fourth Portion of the Duodenum
}

\author{
Behnam Faridian1, Babak Adrangi2, Kiarash Mirkia ${ }^{3}$ \\ ${ }^{1}$ Universidad Autonoma de Guadalajara School of Medicine, Guadalajara, Mexico \\ ${ }^{2}$ Spring Valley Hospital Medical Center, Department of Anesthesiology, Las Vegas, Nevada, USA \\ ${ }^{3}$ Spring Valley Hospital Medical Center, Department of Surgery, Las Vegas, Nevada, USA \\ Email:kmirkia@yahoo.com
}

How to cite this paper: Faridian, B. Adrangi, B. and Mirkia, K. (2018) Perforated Diverticulum of the Fourth Portion of the Duodenum. Open Journal of Emergency Medicine, 6, 1-5.

https://doi.org/10.4236/ojem.2018.61001

Received: February 3, 2018

Accepted: March 17, 2018

Published: March 20, 2018

Copyright (c) 2018 by authors and Scientific Research Publishing Inc. This work is licensed under the Creative Commons Attribution International License (CC BY 4.0).

http://creativecommons.org/licenses/by/4.0/

\begin{abstract}
We are describing a rare occurrence of a perforated diverticulum of the fourth portion of duodenum and surgical repair. Patient expired despite prompt surgical intervention secondary to septic shock, multi-organ-system failure (MOSF) and delayed presentation of the disease.

\section{Keywords}

Duodenum, Perforation, Diverticulum, Fourth Portion, Septic Shock, Sepsis, Multi Organ System Failure
\end{abstract}

\section{Introduction}

Duodenal perforation is still considered a life-threatening condition. Duodenal diverticula incidence can be up to $22 \%$ with approximately $0.03 \%$ having complications [1]. Acquired diverticula are commonly located near the ampulla of Vater in the duodenum. Detection of duodenal diverticula is reported to range from $0.16 \%$ to $6 \%$ in an upper GI examination, $5 \%$ to $27 \%$ during endoscopic retrograde cholangiography (ERCP), and $23 \%$ in an autopsy series [2]. The prevalence increases with age, with the average age ranging from 56 to 76 years old, and occurrence being rare in patients under 40 years of age. The colon is the most common site for gastrointestinal perforations, with the duodenum being the second most common. The majority of diverticula in the third and fourth portion of the duodenum are asymptomatic, while localizing a diverticula in those portions is extremely rare to begin with [3].

In the following case we are reporting a 78 -year-old female who presented with acute onset epigastric and right upper quadrant abdominal pain. She was found to have gallstones with dilation of the common bile duct with a concern 
for choledocholithiasis, without evidence of cholecystitis and pneumoperitoneum specious for perforated peptic ulcer.

\section{Case Report}

A 78-year-old female, with past medical history of hypertension, hyperlipidemia and rheumatoid arthritis, was brought in by Emergency Medical Services as a transfer from another facility. The patient presented to the initial facility with acute onset epigastric and right upper quadrant pain with nausea and vomiting and some shortness of breath. She was found to have gallstones and a dilated common bile duct measuring $10 \mathrm{~mm}$ with a concern for choledocholithiasis without evidence of cholecystitis and pneumoperitoneum specious for perforated peptic ulcer. Patient was transferred to Spring Valley Hospital Medical Center in Las Vegas, NV for further evaluation. She underwent MRCP without contrast that showed a possible mass or inflammatory process in the region of the second portion of the duodenum and pancreatic head, with a possible perforated duodenal ulcer. It also showed trace free fluid around Morison's Pouch, causing obstruction of the distal common bile duct. She underwent a CT scan, which showed a collection of air and debris medial to the second/third portion of the duodenum, along with mild surrounding induration with fluid/induration extending along the right paracolic gutter (Figures 1-3).

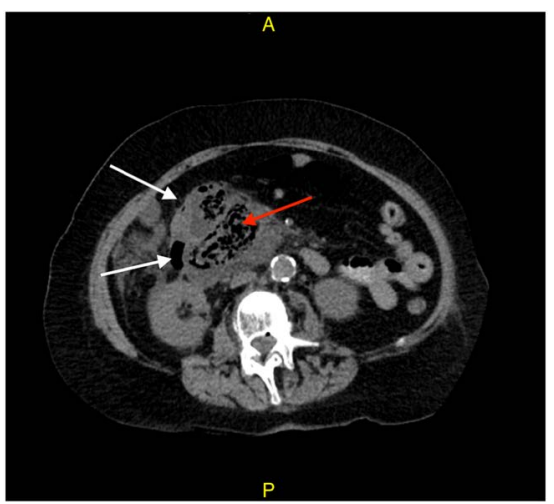

Figure 1. CT scan. Free air (white arrows), Phlegmon (red arrow).

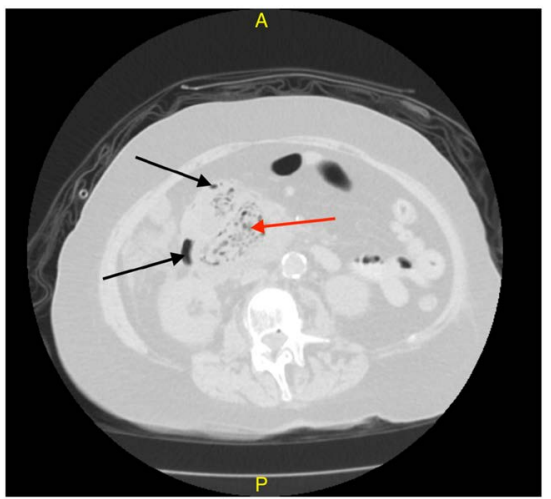

Figure 2. CT scan. Free air (black arrows), Phlegmon (red arrow). 


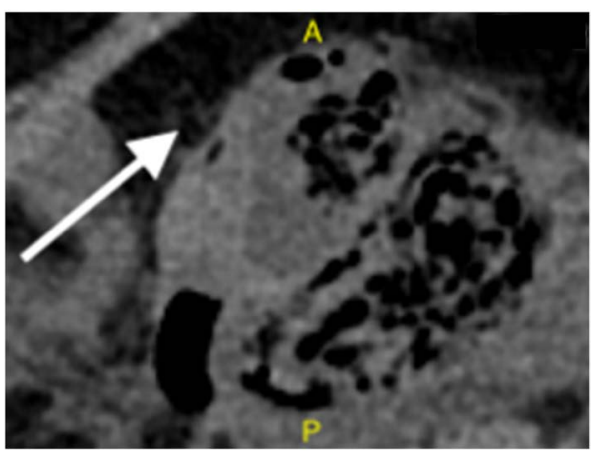

Figure 3. CT scan magnified. Free air (white arrow).

Patient was brought to the operating room emergently for evaluation of this lesion and possible repair of the perforation. An exploratory laparotomy was performed and adhesions were seen from the omentum to the abdominal wall. The posterior stomach was examined and no evidence of perforation was seen. The lesser curvature and lesser sac were entered and the stomach was then followed down to the first and second portion of the duodenum, the adhesions were lysed, finding no evidence of perforation. The third and fourth portions of the duodenum were exposed using a Kocher maneuver, and a duodenal diverticulum was identified at the fourth portion of the duodenum, which had a large perforation. The duodenal diverticulum was stapled off using an Echelon 60 endo-GIA stapler (Figure 4) and the diverticulum removed (Figure 5), with the staple line then being reinforced with 3-0 PDS suture in a running fashion. This was then patched with an omental patch and 3-0 Vicryl sutures.

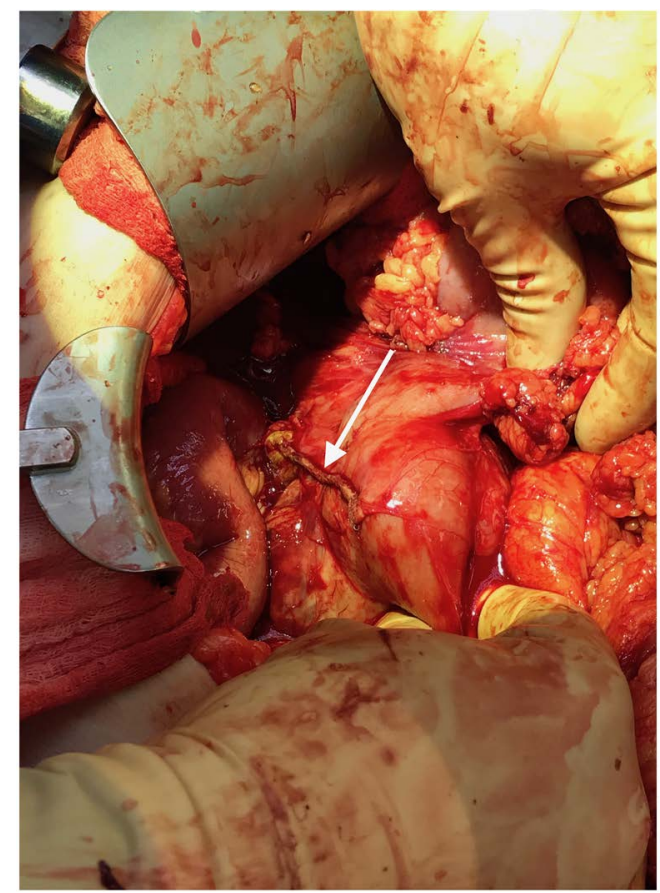

Figure 4. Staple line after removal and repair of perforated duodenal diverticulum. 


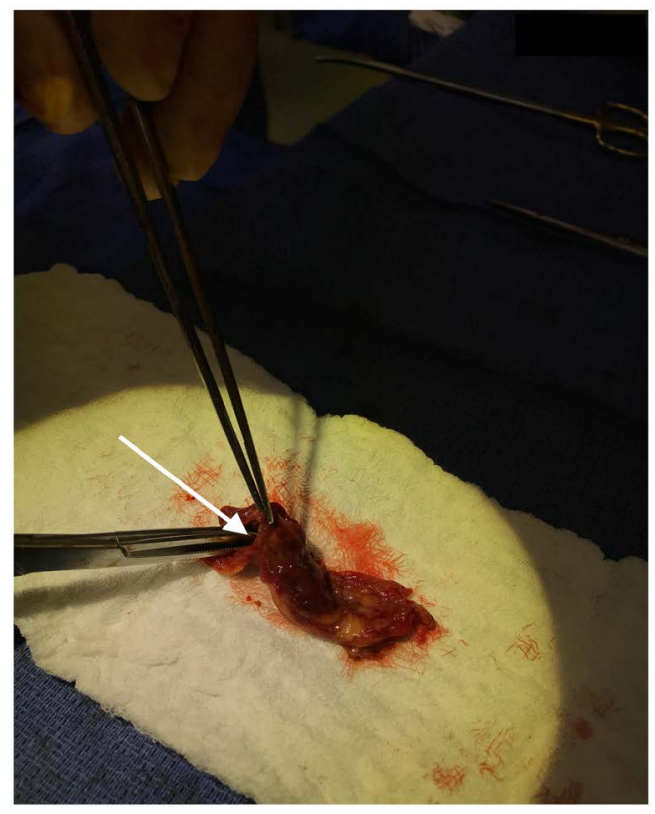

Figure 5. Diverticulum specimen with perforation (white arrow).

Upon arrival to the operating room at Spring Valley, the patient was showing signs of diffuse skin mottling and septic shock. The patient's liver function tests were normal. The initial facility found patient's lipase to be 713, white blood cell count of 14.7, and creatinine of 1.2. Post surgically, the creatinine increased to 1.6 with a metabolic acidosis with $\mathrm{pH}$ of 7.23. Patient also continued to be hypotensive, requiring vasopressors. The creatinine continued to rise to 3.8 and renal failure followed requiring Continuous Renal Replacement Therapy (CRRT) via her right femoral vein dialysis catheter. While undergoing CRRT, platelets were found to have dropped down to 41,000 and patient was transfused with platelets. The family withdrew care postoperative day 4 and she expired with her family at bedside.

\section{Discussion}

In this report we are describing a duodenal diverticulum perforation that occurred in an area not commonly seen for diverticula, along with perforation that can result in sepsis and death. The first reported case of duodenal diverticula occurred in 1710 by Dr. Pierre Jean Baptiste Chomel, a French pathologist [4]. Only 101 cases of duodenal diverticula were reported by 1989, with less than 200 reported to date, with the vast majority being in the first and second portion of the duodenum. Perforation of such diverticula is the rarest and the most serious complication, with just over 100 cases reported to date. Early preoperative diagnosis has proven to be difficult because the symptoms presented by the patient are nonspecific. Most discovered diverticula are incidental on imaging or during surgery. However, they are frequently mistaken for pancreatic pseudocysts and fluid collections, periampullary neoplasm, or biliary cysts. Early diagnosis of 
perforations is important for survival, as some statistics show a $65 \%$ or higher mortality rate when the diagnosis is delayed for 24 hours or more. On the other hand, those who receive surgical treatment within the first 24 hours had about a 5\% mortality rate [5]. Perforations can lead to septic shock, a life-threatening condition, and can rapidly deteriorate into multi-organ system failure. In this case, it is unknown if the perforation was present at initial presentation or if the perforation occurred after, starting with a micro perforation. The dilation of the common bile duct could have been caused, not by a gallstone, but by the free fluid in Morison's Pouch causing obstruction of the duct. This free fluid could have been from the perforation. A crucial diagnostic clue has been found to be periduodenal or retroperitoneal free air [6]. An upper gastrointestinal series with contrast media collected in the diverticulum with extravasation can be accurate for diagnosis of the perforation also [7].

\section{Conclusion}

There are few cases of diverticula of the fourth portion of the duodenum reported, and even fewer cases of perforations of such diverticula. While it is recommended that asymptomatic diverticula left alone, early diagnosis and treatment of a perforated diverticulum are imperative. Proper diagnosis, immediate surgical intervention and antibiotic therapy can drastically reduce the mortality rate.

\section{Conflict of Interest}

None of the authors have any financial or personal relationships that could influence this work.

\section{References}

[1] de Perrot, T., Poletti, P.A., Becker, C.D. and Platon, A. (2012) The Complicated Duodenal Diver-Ticulum: Retrospective Analysis of 11 Cases. Clinical Imaging, 36, 287-294. https://doi.org/10.1016/i.clinimag.2011.11.007

[2] Schwartz, S.I. and Brunicardi, F.C. (2015) Schwartz's Principles of Surgery. 10th Edition, McGraw Hill Medical, New York, N.Y.

[3] Avalos-González, J. and Zaizar-Magaña, A. (2008) Duodenal Diverticulum in the Third Portion of Duodenum as a Cause of Upper Gastrointestinal Bleeding and Chronic Abdominal Pain. Case Report and Literature Review. Cirugia y cirujanos 76, 65-69.

[4] Chomel, J. (1710) Report of a Case of Duodenal Diverticulum Containing Gallstones. Histoire de 1 Académie Royale des Sciences, 1710, 48-50.

[5] Roman, E., Silva, Y. and Lucas, C. (1971) Management of Blunt Duodenal Injury. Surg Gynecol Obstet, 132, 7-14.

[6] Glasser, C.M., Goldman, S.M., Roda, C.L., et al. (1978) Preoperative Diagnosis of a Perforated Duodenal Diverticulum. American Journal of Roentgenology, 130 , 563-564. https://doi.org/10.2214/ajr.130.3.563

[7] Sakurai, Y., Miura, H., Matsubara, T., et al. (2004) Perforated Duodenal Diverticulum Successfully Diagnosed Preoperatively with Abdominal CT Scan Associated with Upper Gastrointestinal Series. Journal of Gastroenterology, 39, 379-383. https://doi.org/10.1007/s00535-003-1306-5 